\title{
Reproductive Immunology a New Platform for the Dissemination of Research Outputs
}

\section{Sharon McCracken*}

Division of Perinatal Research, Kolling Institute of Medical Research, Royal North Shore Hospital, Reserve Rd, St Leonards, NSW 2065, Australia

${ }^{*}$ Corresponding author: Sharon McCracken, Division of Perinatal Research, Kolling Institute of Medical Research, Royal North Shore Hospital, Reserve Rd, St Leonards, NSW 2065, Australia, Tel: +61 29926 4832; Email: Sharon.mccracken@sydney.edu.au

Received date: March 08, 2016; Accepted date: March 10, 2016; Published date: March 14, 2016

Citation: McCracken S (2016) Reproductive Immunology a New Platform for the Dissemination of Research Outputs. Reproductive Immunol Open Acc 1:6. doi: 10.21767/2476-1974.10006

Copyright: (C) 2016, McCracken S. This is an open-access article distributed under the terms of the Creative Commons Attribution License, which permits unrestricted use, distribution, and reproduction in any medium, provided the original author and source are credited.

\section{Dear Authors and readers}

We the Editorial team of Reproductive Immunology: Open Access welcomes all eminent Authors and readers to the launch of the new journal, Reproductive Immunology: Open Access (RIOA). This is a peer reviewed open access journal and the main aim of the journal is to highlight the new aspects of immunological concepts related to the reproductive system and reproduction. While our main focus is Reproductive immunology our journal encapsulates research that focus on extending and advancing our understanding of immunological concepts in general.

RIOA provides a platform for the dissemination of research outputs that may result in potential solutions or treatments for infertility, recurrent pregnancy loss or pathological placentation that result in complications such as preeclampsia or intra uterine growth restriction. Reproductive Immunology is a facet of medicine that pertains to the interfaces between the immune system and the reproductive system which includes maternal immune tolerance towards the foetus and immunological interactions across the blood-testis barrier.
There is much recognition that the immune system plays a significant role in promoting healthy pregnancy and conversely in preventing healthy pregnancies. The main aim of this Journal of Reproductive Immunology is to provide the ideal platform for the dissemination of data from high quality research in all aspects pertaining to reproductive. As such RIOA will incorporate research into Gametogenesis and Embryogenesis, Implantation and Placental Development, Gestation and Parturition, Mammary Gland and Lactation. In addition we welcome research into understanding Autoimmunity, Mucosal Immunology, Immunoendocrinology, Reproductive Immunotherapies, Developmental Immunology as well as Immunology of Reproductive Cancers.

Finally we would like to encourage Authors and readers to contribute to the scope of this journal by submitting their research such that we may enhance the knowledge of Reproductive Immunology globally. 